\title{
A within-sibling pair analysis of lifestyle behaviours and BMI z-score in the multi-centre I.Family study
}

\author{
L.H. Bogl a, ${ }^{\text {*,1 }}$, K. Mehlig ${ }^{\mathrm{b}, 1}$, T. Intemann ${ }^{\mathrm{a}, \mathrm{c}}$, G. Masip ${ }^{\mathrm{d}}$, A. Keski-Rahkonen ${ }^{\mathrm{d}}$, P. Russo ${ }^{\mathrm{e}}$, \\ N. Michels ${ }^{f}$, L. Reisch ${ }^{g}$, V. Pala ${ }^{\mathrm{h}}$, L. Johnson ${ }^{\mathrm{i}}$, D. Molnár ${ }^{\mathrm{j}}$, M. Tornaritis ${ }^{\mathrm{k}}$, \\ T. Veidebaum ${ }^{1}$, L. Moreno ${ }^{\mathrm{m}}$, W. Ahrens ${ }^{\mathrm{a}, \mathrm{c}}$, L. Lissner ${ }^{\mathrm{b}}$, J. Kaprio ${ }^{\mathrm{d}, \mathrm{n}, 1}{ }^{\text {, A. Hebestreit }}{ }^{\mathrm{a}, 1}$ on \\ behalf of the I.Family Consortium
}

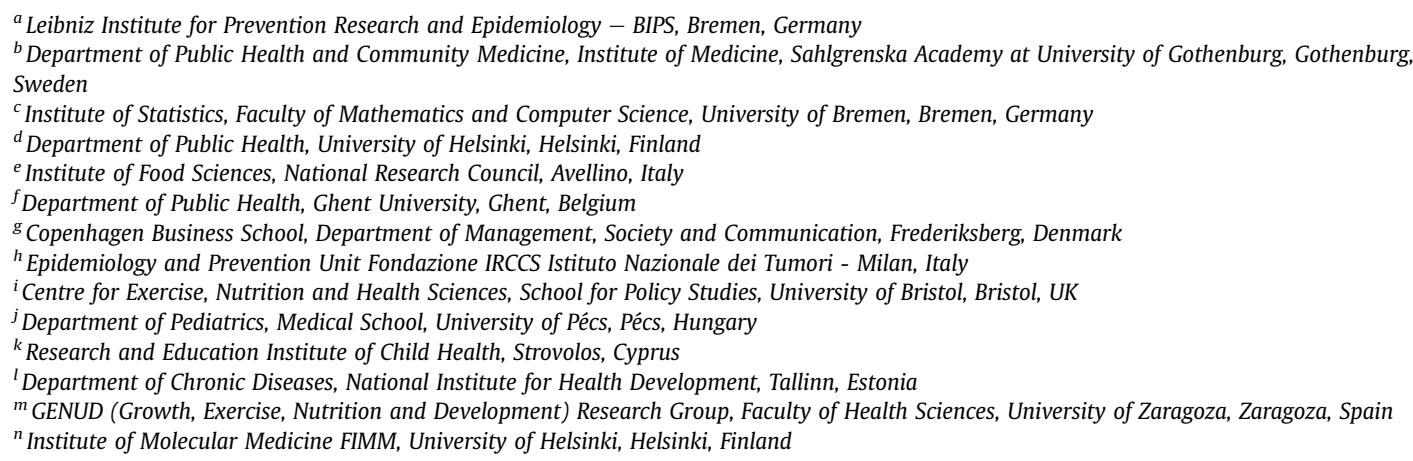

Received 1 November 2018; received in revised form 15 January 2019; accepted 30 January 2019

Handling Editor: P. Strazzullo

Available online

\section{KEYWORDS \\ Overweight- \\ discordant; \\ Sibling pairs; \\ Twin pairs; \\ Body mass index; \\ Energy intake; \\ MVPA}

\begin{abstract}
Background and aims: By investigating differences in lifestyle behaviours and BMI in sibling pairs, family-level confounding is minimized and causal inference is improved, compared to cross-sectional studies of unrelated children. Thus, we aimed to investigate within-sibling pair differences in different lifestyle behaviours and differences in BMI z-scores in children and adolescents.

Methods and results: We examined three groups of sibling pairs 1$)$ all same-sex sibling pairs with maximum 4 years age difference $(\mathrm{n}=1209$ pairs from 1072 families in 8 countries, mean age 10.7 years, standard deviation 2.4 years), 2) sibling pairs discordant for overweight $(\mathrm{n}=262$ ) and 3 ) twin pairs $(\mathrm{n}=85)$. Usual dietary intake was estimated by 24 -h recalls and time spent in light (LPA) and moderate-to-vigorous physical activity (MVPA) was measured by accelerometers. Screen time, sleep and dieting for weight loss were assessed by questionnaires.

Within all 3 groups of sibling pairs, more time in MVPA was associated with lower BMI z-score. Higher energy intake was associated with higher BMI z-score within twin pairs and within all
\end{abstract}

Abbreviations: 24HDR 24-hour dietary recall, BMI Body mass index; IOTF International Obesity Task Force, ISCOLE International Study of Childhood Obesity; Lifestyle and the Environment, MVPA Moderate-to-vigorous physical activity; PA Physical activity, SACANA SelfAdministered Children; Adolescents and Adult Nutrition Assessment, SACINA Self-Administered Children and Infant Nutrition Assessment; TV Television, WC Waist circumference.

* Corresponding author. Leibniz Institute for Prevention Research and Epidemiology-BIPS GmbH, Achterstrasse 30, D-28359, Bremen, Germany.

E-mail addresses: bogl@leibniz-bips.de (L.H. Bogl), kirsten.mehlig@gu.se (K. Mehlig), intemann@leibniz-bips.de (T. Intemann), guiomar.masipmanuel@helsinki.fi (G. Masip), anna.keski-rahkonen@helsinki.fi (A. Keski-Rahkonen), paola.russo@isa.cnr.it (P. Russo), Nathalie.Michels@UGent.be (N. Michels), lre.msc@cbs.dk (L. Reisch),Valeria.Pala@istitutotumori.mi.it (V. Pala), Laura.Johnson@bristol.ac.uk (L. Johnson), denes.molnar@aok. pte.hu (D. Molnár), tor.michael@cytanet.com.cy (M. Tornaritis), toomas.veidebaum@tai.ee (T. Veidebaum), lmoreno@unizar.es (L. Moreno), ahrens@leibniz-bips.de (W. Ahrens), lauren.lissner@gu.se (L. Lissner), jaakko.kaprio@helsinki.fi (J. Kaprio), hebestr@leibniz-bips.de (A. Hebestreit).

${ }^{1}$ These authors contributed equally to this work. 
sibling pairs who were not currently dieting for weight loss. Regarding LPA, screen time or sleep duration, no or inconsistent associations were observed for the three groups of sibling pairs. Conclusions: MVPA and energy intake were associated with BMI differences within sibling and twin pairs growing up in the same home, thus independent of family-level confounding factors. Future studies should explore whether genetic variants regulating appetite or energy expenditure behaviours account for weight differences in sibling pairs.

(c) 2019 IAgrE. Published by Elsevier Ltd. All rights reserved.

\section{Introduction}

Child and adolescent overweight and obesity are major health problems with numerous negative health consequences. These include immediate physiological [1] and psychological consequences [2], as well as an adverse impact on adult health [3]. Obesity tracks in families [4], and arises from a combination of genetic and environmental factors that interact to promote the overweight phenotype [5]. Twin and family studies suggest that genetic factors account for a large proportion of individual differences in body weight and obesity, with the remaining variance explained by shared and unique environmental factors [6,7].

Both energy intake and energy expenditure related behaviours need to be considered as possible proximal risk factors of childhood obesity. The multi-national crosssectional International Study of Childhood Obesity, Lifestyle and the Environment (ISCOLE) has identified moderate-to-vigorous physical activity (MVPA), sleep duration and television (TV) viewing as important correlates of childhood obesity among 9-11 year old children [8]. The European multi-centre IDEFICS (Identification and prevention of dietary- and lifestyle-induced health effects in children and infants) and I.Family studies examined children aged 2-17 years and indicated energy intake [9], sleep duration [10], TV viewing [11] and physical activity (PA) [12] as potential correlates of anthropometric markers or risk factors for childhood overweight.

Most previous investigations studied individual children, requiring adjustment for a range of measured familial confounding factors such as socioeconomic status or maternal weight status. Despite the fact that siblings share a substantial part of their family environment and genetic variability - on average, $50 \%$ of their segregating genes lifestyle behaviours may be non-shared between siblings. The family-based recruitment in I.Family allows us to investigate the association between lifestyle behaviours and body mass index (BMI) $z$-scores within sibling pairs and to explore which behaviours distinguish same-sex sibling pairs discordant for overweight. The advantage of the sibling design is that it inherently controls for a range of measured but also unmeasured familial factors that same-sex siblings share [13], such as parental characteristics, family size, gender-specific socialisation, family and neighbourhood food environment, or shared friends. Twin pairs, as a special case of sibling pairs share additional factors, such as age, maternal age at birth or maternal food intake during pregnancy, and monozygotic twins are genetically identical at the DNA sequence level. Thus, the aim of the present study was to investigate associations between lifestyle behaviours and BMI $z$-score within sibling pairs, therefore minimizing family-level confounding compared to studies of unrelated children.

\section{Methods}

\section{Participants}

The sibling pairs were participants of the I.Family study and were examined in 2013/2014. The families were recruited from Belgium, Cyprus, Estonia, Germany, Hungary, Italy, Spain, and Sweden [14]. Ethics approval was obtained from responsible committees in each country in accordance with the ethical standards of the 1964 Declaration of Helsinki and its later amendments. All parents or legal guardians of the participating minor children gave written informed consent. Children older than 16 years provided written informed consent, while children aged 12 and over gave a simplified written consent. Younger children gave oral consent for examinations and sample collection. Children could consent to single components of the study while abstaining from others.

\section{Measurements}

\section{Examinations}

Anthropometric parameters were measured in fasting condition and light clothing (underwear, T-shirts). Weight was measured using an electronic scale (Tanita BC $418 \mathrm{MA}$ scale and a prototype of the TANITA BC 420 MA scale for children under 6 years with smaller feet; TANITA Europe $\mathrm{GmbH}$, Sindelfingen, Germany). Height was measured barefoot and to the nearest $0.1 \mathrm{~cm}$ using a Seca 225 or Seca 213 stadiometer (Seca GmbH \& KG, Birmingham, UK) in accordance with international standards for anthropometric assessment and weight $(\mathrm{kg})$ [15]. Waist circumference (WC) was measured to the nearest $0.1 \mathrm{~cm}$ in upright position with relaxed abdomen at the midpoint between the lowest rib margin and the iliac crest) [15]. BMI was calculated by dividing body weight in kilograms by body 
height squared. Age and sex-specific BMI z-scores were computed according to Cole et al. [16]. Weight status was defined as recommended by the extended International Obesity Task Force (IOTF) cut-offs for thinness, overweight and obesity in children [17].

\section{Questionnaire and interview data}

The questionnaires were originally developed in English and translated into local languages. The quality of translations was checked by back-translations. Parents or guardians completed a "child questionnaire" for children below the age of 12 years. Children 12 years or older completed a self-administered "teen questionnaire". Information on dieting was assessed by asking whether the child is currently on a weight loss diet (Yes vs. No). Information on screen time was assessed separately for weekend and weekdays by asking how many hours per day (hrs/d) the child usually spends watching television (including videos or DVDs) and/or sitting in front of a computer or game console. Screen time was assessed as the sum of hours spent in front of a television and/or computer. Daily sleep duration was calculated by summing up the hours of nocturnal sleep duration and napping duration (hrs/d). Screen time and sleep were weighted to account for weekday and weekend values. Age at menarche and voice mutation status were self-reported in children 8 years and older (pre-pubertal or pubertal) and classified as pubertal if menarche has already occurred in girls or if voice alterations have already started or were completed in boys (Yes vs. No). The parent or legal guardian took part in an interview on kinship and household composition using Computer Assisted Telephone Interviewing, Computer Assisted Personal Interviewing, face-to-face interview or pen-and-paper versions. The interview inquired about the relatedness of all persons living in the same household.

\section{Accelerometer data}

Physical activity was objectively measured by uniaxial accelerometry using Actigraph models (GT1M and ActiTrainer; Actigraph, Pensacola, Florida, USA) [18]. The accelerometers were attached to the right hip with an elastic belt. Each child was verbally instructed to wear the device for at least 3 days during waking hours, except when showering or swimming. Parents were given written instructions on how to use the accelerometer and were asked to complete diaries to record non-wear times of the device. Time spent in MVPA is based on cleaned accelerometer data that only contains measurements that have passed the minimum inclusion criteria of at least $8 \mathrm{~h}$ wear-time per day for at least 3 days. The sample interval was set to an epoch of $15 \mathrm{~s}$. Non-wear time was defined as $20 \mathrm{~min}$ or more of consecutive zero counts. The average activity level of the children was defined by counts per minute (cpm). The average activity level of the children was defined by counts per minute (cpm). Light PA was defined as $>100$ and $<2296 \mathrm{cpm}$ and MVPA was defined as $>2296 \mathrm{cpm}$ based on the cut-off values for school-aged children proposed by Evenson et al. [19]. The cumulative duration for MVPA was expressed as minutes per day.

\section{Dietary intake data}

Dietary intake was assessed using a 24-hour dietary recall (24HDR) assessment program, called 'Self-Administered Children, Adolescents and Adult Nutrition Assessment' (SACANA) [20,21], a web based instrument based on the validated 'Self-Administered Children and Infant Nutrition Assessment' (SACINA) offline version [22]. SACANA is an interactive tool that assesses information on amount and type of all foods and drinks that were consumed during the previous day, starting with the first intake after waking up in the morning and ending with the last intake prior to going to sleep. Standardized food images were used to assist portion size estimation as well as probing questions regarding usual combinations of foods such as bread and bread spreads. Country-specific food composition tables (FCT) were used to match simple foods or European homogeneous multi-ingredient foods typically consumed in the different countries [23]. Children 11 years and above completed a self-administered 24HDR whereas parents were asked to proxy report the intake for younger children and/or assist children in filling in the 24HDR [24]. The participants were encouraged to complete repeated 24HDRs, including two weekdays and one weekend day, but the actual availability of 24HDRs varied among children between 1 and 12 days (mean number of days $=1.9$ days). Each recall day was classified as under-reported, plausibly reported and over-reported energy intake according to age- and sex-specific Goldberg cut-offs [25] adapted for children [26]. In the following, misreporting refers to both under- and over-reporting of total energy intake.

For the whole study population and for the subjects with plausible reports of energy intake, the individual usual energy intake was estimated based on the validated $\mathrm{NCI}$ method separately for boys and girls $[27,28]$. The advantage of the $\mathrm{NCI}$ method is its ability to take into account the intraindividual variation following a skewed distribution and to incorporate covariates. All models considered the day of the week, the interview sequence, age and country as covariates. For more details on the specifications of the models for the usual intake estimation see Hebestreit et al. [29].

\section{Inclusion criteria for sibling pairs}

Figure 1 provides details of our sample of siblings and of both subsamples. From the 4298 sibling pairs in I.Family, we excluded opposite-sex sibling pairs $(n=2110)$ because the focus on same-sex siblings controls for gender differences in biological and social characteristics that are ubiquitous and controlling for these by design brings more power to the analyses. We further excluded same-sex sibling pairs with an age difference greater than 4 years ( $\mathrm{n}=784$ ), leaving 1404 same-sex sibling pairs with a maximum age difference of 4 years. Since there were 116 pairs with missing data on all lifestyle behaviours (screen time, sleep, accelerometry and 24HDR) and 79 pairs with 


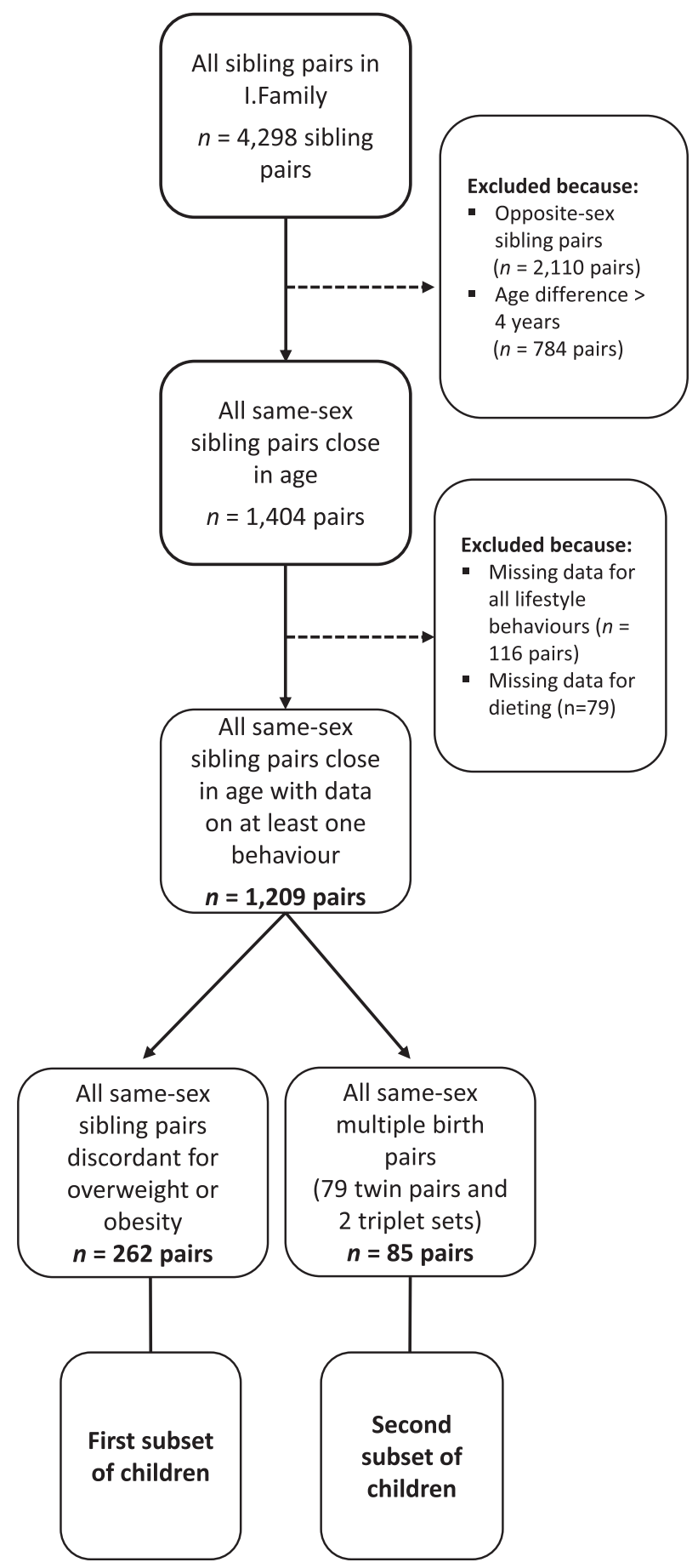

Figure 1 Flow chart depicting the identification of all same-sex sibling pairs in the I.Family study and two subsets of these children (overweight-discordant sibling pairs and twin pairs).

missing dieting information, the final sample for the analysis included 1209 same-sex sibling pairs (53\% boyboy pairs). Among these, we identified two subsets of children 1) siblings pairs discordant for overweight $(\mathrm{n}=262)$ and 2) 79 twin pairs and 2 triplet sets $(\mathrm{n}=85$ multiple birth pairs). Twins were identified by the same birth dates, and therefore include a mixture of monozygotic and dizygotic twins because zygosity information is not available. In case that there was more than one discordant sibling pair for overweight in a family, we retained the most discordant pair. Overweight discordance was defined as one sibling being overweight or obese and the other being underweight or normal-weight as previously defined $[30,31]$. Throughout the manuscript, discordance for overweight will therefore include obesity if applicable. However, because this definition may include sibling pairs where one sibling meets the definition for overweight and his/her sibling does not but is on the borderline for overweight, we added an arbitrary minimum difference of at least $10 \mathrm{BMI}$ percentiles between the sibling pairs. As it was possible for children to opt out from single examination modules, the actual number of sibling pairs varied for the different lifestyle behaviours. The number of sibling pairs with available data for screen time, sleep, accelerometry and 24HDR is shown in Table 1.

\section{Statistical analysis}

Basic characteristics are shown for all sibling pairs, sibling pairs discordant for overweight and twin pairs separately. Each individual in a pair of siblings or twins was randomly assigned as sibling 1 or sibling 2 . The within-pair differences in lifestyle behaviours and BMI z-scores were calculated by subtracting the value of one sibling from that of the other sibling (i.e. value of sibling 1 minus value of sibling 2). Mixed linear regression was used to examine the association between within-pair differences in lifestyle behaviours and differences in BMI z-scores adjusting for sex, differences in age, and differences in height. Because the analysis of all sibling pairs may include more than one sibling pair per family, these models were further adjusted for family as a random effect. A positive association of within-pair differences means that children with higher values for a lifestyle behaviour (e.g. energy intake) also have higher BMI z-scores compared to their sibling, while a negative association of within-pair differences means that children with higher values for a lifestyle behaviour (e.g. MVPA) have lower BMI z-scores than their respective sibling.

We carefully evaluated the energy intake-BMI association. First, we analysed the energy intake-BMI association among all sibling pairs who reported their energy intakes. Next, we repeated the same model with additional adjustment for dieting/misreporting status of the sibling pairs. Finally, we repeated the analysis only among sibling pairs where both siblings were not currently dieting and then among those who had plausible reports of energy intake. Dieting status of the sibling pairs was coded as follows: " 0 " if both siblings were not currently dieting, " 1 " if sibling 1 was currently dieting and sibling 2 not, " 2 " if sibling 2 was currently dieting and sibling 1 not, or " 3 " if both siblings were currently dieting. Misreporting of the sibling pairs was coded as " 0 " if energy reports of both siblings were implausible, " 1 " if sibling 1 was implausible, " 2 " if sibling 2 was implausible and " 3 " if energy reports of both siblings were plausible.

We tested the effect of further adjustment for mean age and mean BMI of the siblings, and for differences in 
Table 1 General characteristics of the sibling pair groups.

\begin{tabular}{|c|c|c|c|}
\hline & All sibling pairs & $\begin{array}{l}\text { Sibling pairs discordant } \\
\text { for overweight }\end{array}$ & Twin pairs $^{\mathrm{a}}$ \\
\hline \multicolumn{4}{|l|}{ Country (n, \%) } \\
\hline Italy & $180(14.9)$ & $48(18.3)$ & $9(10.6)$ \\
\hline Estonia & $127(10.5)$ & $16(6.1)$ & $13(15.3)$ \\
\hline Cyprus & $343(28.4)$ & $94(35.9)$ & $24(28.4)$ \\
\hline Belgium & $58(4.8)$ & $6(2.3)$ & $1(1.2)$ \\
\hline Sweden & $110(9.1)$ & $9(3.4)$ & $4(4.7)$ \\
\hline Germany & $174(14.4)$ & $41(15.7)$ & $17(20.0)$ \\
\hline Hungary & $157(13.0)$ & $35(13.4)$ & $8(9.4)$ \\
\hline Spain & $60(5.0)$ & $13(5.0)$ & $9(10.6)$ \\
\hline \multicolumn{4}{|l|}{ BMI z-score data } \\
\hline Number of sibling pairs & 1209 & 262 & 85 \\
\hline Number of boy and girl pairs & $639 / 570$ & $131 / 131$ & $43 / 42$ \\
\hline Number of families & 1072 & 262 & 79 \\
\hline Mean age, years (min, max) & $10.7(3.2,17.1)$ & $10.9(3.8,16.7)$ & $10.7(3.2,15.9)$ \\
\hline Mean BMI z-score (min, max) & $0.45(-3.50,3.75)$ & $1.07(-0.74,2.28)$ & $0.15(-2.22,3.07)$ \\
\hline Mean BMI percentile (min, max) & $0.61(0.00,1.00)$ & $0.78(0.47,0.94)$ & $0.53(0.02,1.00)$ \\
\hline \multicolumn{4}{|l|}{ Screen time data } \\
\hline Number of sibling pairs & 1116 & 240 & 79 \\
\hline Mean screen time, hrs/d (min, max) & $2.2(0.04,8)$ & $2.4(0.2,8)$ & $2.0(0.07,6.2)$ \\
\hline \multicolumn{4}{|l|}{ Sleep data } \\
\hline Number of sibling pairs & 1153 & 249 & 81 \\
\hline Mean sleep duration, hrs/d (min, max) & $9.5(5.8,15.5)$ & $9.4(5.8,15.5)$ & $9.5(7.9,12.5)$ \\
\hline \multicolumn{4}{|l|}{ Accelerometer data } \\
\hline Number of sibling pairs & 476 & 103 & 37 \\
\hline Mean accelerometer wear time, hrs/d (min, max) & $12.4(8.3,18.3)$ & $12.4(9.0,15.8)$ & $12.2(10.6,14.0)$ \\
\hline Mean time spent in MVPA, min/d (min, max) & $39(3,117)$ & $35(5,116)$ & $36(3,71)$ \\
\hline Mean time spent in LPA, min/d (min, max) & $323(147,502)$ & $317(185,457)$ & $308(166,420)$ \\
\hline \multicolumn{4}{|l|}{ 24-h dietary recall data } \\
\hline \multicolumn{4}{|l|}{ Pairs with reported energy intake } \\
\hline Number of sibling pairs & 491 & 90 & 42 \\
\hline Mean energy intake, $\mathrm{kcal} / \mathrm{d}(\min , \max )$ & $1629(888,2453)$ & $1627(937,2453)$ & $1590(888,2277)$ \\
\hline \multicolumn{4}{|l|}{ Pairs with plausible reported energy intake } \\
\hline Number of sibling pairs & 361 & 63 & 28 \\
\hline Mean energy intake, $\mathrm{kcal} / \mathrm{d}(\min , \max )$ & $1799(1351,2424)$ & $1843(1422,2370)$ & $1824(1352,2299)$ \\
\hline
\end{tabular}

pubertal status. Because interactions of main exposures with sex of the sibling pair were not significant, all results are presented for boy-boy and girl-girl sibling pairs combined. In the overall sample, sensitivity analysis excluding half- and stepsiblings and/or multiple birth pairs did not change the effect estimates. All mixed models were carried out among all same-sex siblings close in age and within that group the following two subsets 1) sibling pairs discordant for overweight and 2) twin pairs. All analyses were performed with Stata 13.1 (Stata Corporation, College Station, TX, USA http://www.stata.com).

\section{Results}

\section{Descriptive characteristics of the sibling pair groups}

The general characteristics of all same-sex sibling pairs, sibling pairs discordant for overweight and twin pairs separately is shown in Table 1. Approximately half of the sibling pairs were boy-boy pairs and about half were girlgirl pairs. Most sibling pairs were identified in Cyprus, Italy and Germany. Cyprus also displayed the largest number of overweight-discordant sibling pairs and twin pairs. On average, the overweight-discordant siblings pairs tended to have a higher and the twin pairs a lower BMI z-score than all sibling pairs. Mean accelerometer wear-time was $12.2-12.4 \mathrm{~h}$ per day in the sibling pair groups.

Out of the 1209 sibling pairs, 981 (81\%) were concordant for dieting status and 228 (19\%) were discordant for dieting status. Only 39 pairs (3\%) were such that both siblings were currently on a weight loss diet. Out of the 491 sibling pairs with 24HDR data, 295 (60\%) pairs reported plausible energy intakes, 58 (11.8\%) were concordant for misreporting and 138 (28\%) were discordant for misreporting, of which the vast majority (131) was discordant for underreporting. Over-reporting was negligible and only reported by 7 siblings in total.

\section{Overweight-discordant pairs}

Among the 262 sibling pairs discordant for overweight, most pairs were non-overweight vs. overweight sibling pairs $(n=194)$, whereas fewer pairs were composed of normal-weight vs. obese sibling pairs $(\mathrm{n}=60)$. A minority of leaner siblings were underweight with an overweight sibling $(\mathrm{n}=8)$. Among the 262 sibling pairs discordant for 
Table 2 Linear mixed models for the associations of within-pair differences $(\Delta)$ in lifestyle behaviours and $\Delta$ BMI z-score (outcome) within 3 sibling pair groups.

\begin{tabular}{|c|c|c|c|c|c|}
\hline Independent variables (separate models) & $\mathrm{n}$ & $\beta$ & SE & $95 \% \mathrm{CI}$ & P-value \\
\hline \multicolumn{6}{|l|}{$\Delta$ Screen time, hrs/d } \\
\hline All sibling pairs & 1116 & 0.08 & 0.02 & $0.03,0.12$ & 0.002 \\
\hline Sibling pairs discordant for overweight & 240 & 0.13 & 0.07 & $0.00,0.27$ & 0.055 \\
\hline Twin pairs ${ }^{\mathrm{a}}$ & 79 & -0.09 & 0.07 & $-0.23,0.04$ & 0.168 \\
\hline \multicolumn{6}{|l|}{$\Delta$ Sleep duration, hrs/d } \\
\hline All sibling pairs & 1153 & -0.06 & 0.03 & $-0.12,0.01$ & 0.077 \\
\hline Sibling pairs discordant for overweight & 249 & -0.11 & 0.08 & $-0.27,0.05$ & 0.185 \\
\hline Twin pairs $^{\mathrm{a}}$ & 81 & 0.21 & 0.16 & $-0.10,0.52$ & 0.182 \\
\hline \multicolumn{6}{|l|}{$\Delta$ Time spent in MVPA, $10 \mathrm{~min} / \mathrm{d}$} \\
\hline All sibling pairs & 476 & -0.05 & 0.02 & $-0.09,-0.00$ & 0.034 \\
\hline Sibling pairs discordant for overweight & 103 & -0.23 & 0.08 & $-0.37,-0.08$ & 0.003 \\
\hline Twin pairs $^{\mathrm{a}}$ & 37 & -0.07 & 0.02 & $-0.11,-0.02$ & 0.002 \\
\hline \multicolumn{6}{|l|}{$\Delta$ Time spent in LPA, $10 \mathrm{~min} / \mathrm{d}$} \\
\hline All sibling pairs & 476 & 0.01 & 0.01 & $-0.01,0.03$ & 0.253 \\
\hline Sibling pairs discordant for overweight & 103 & -0.01 & 0.03 & $-0.07,0.05$ & 0.663 \\
\hline Twin pairs $^{\mathrm{a}}$ & 37 & -0.01 & 0.02 & $-0.05,0.02$ & 0.418 \\
\hline
\end{tabular}

The model is adjusted for sex, within-pair differences in age and within-pair differences in height. Country and family are included as random effects, if applicable.

Bold P-values indicate significant at $\mathrm{p}<0.05$.

Abbreviations: BMI, body mass index; $\mathrm{n}$, number of sibling pairs; $\beta$, regression coefficient; SE, standard error; $\mathrm{CI}$, confidence interval; MVPA, moderate-to-vigorous physical activity; LPA, light physical activity.

${ }^{a}$ Including 6 pairs from triplets.

overweight, 129 pairs had an older overweight sibling, 124 pairs had a younger overweight sibling and 9 pairs were twin pairs.

BMI z-score was by definition higher in siblings with overweight (mean \pm SD: $1.89 \pm 0.45$ ) than in their nonoverweight (mean \pm SD: $0.24 \pm 0.63$ ) siblings. Overweight siblings tended to be taller (mean \pm SD: $148 \pm 17.1 \mathrm{~cm}$ ) than their non-overweight siblings (mean \pm SD: $145 \pm 17.9 \mathrm{~cm}$ ). Age did not differ between siblings discordant for overweight (mean \pm SD: $10.9 \pm 0.2$ years for both siblings). Mean screen time per day was 2.5 (SD: 1.5) hours per day in siblings with overweight and 2.3 (SD: 1.4) hours per day in their non-overweight siblings. Mean time spent in MVPA was 32 min (SD: 19.3) and 39 (SD: 26.4) minutes per day in overweight and non-overweight siblings, respectively. Mean energy intake per day was 1639 (SD: 323) kcal in siblings with overweight and 1614 (SD: $310) \mathrm{kcal}$ in their non-overweight sibling. A substantial proportion (37\%) of the siblings with overweight reported to be currently on a weight loss diet, while only $11 \%$ of the non-overweight siblings were currently dieting. Neither the leaner nor the heavier siblings within overweightdiscordant pairs over-reported their energy intakes.

\section{Lifestyle behaviours and BMI $z$-score differences in the sibling pair groups}

Table 2 shows the associations between the different lifestyle behaviours and BMI z-score differences within the 3 groups of sibling pairs adjusted for sex, differences in height and differences in age. Higher screen time was associated with higher BMI z-scores within all sibling pairs. Within-pair differences in screen time were not related to differences in BMI z-score within overweightdiscordant sibling pairs or twin pairs, however, $41 \%$ of the twin pairs did not differ in screen time (Table 2). Sleep duration was not significantly related to BMI-z scores differences within any of the 3 sibling groups. More time spent in MVPA was associated with lower BMI z-score within all 3 groups of sibling pairs.

Among all reporters of energy intake, higher energy intake was related to higher BMI z-score only within twin pairs. After adjustment for misreporting status of the sibling pairs, higher energy intake was related to higher BMI z-score within all 3 groups of sibling pairs. Energy intake was related to higher BMI z-score within all sibling and twin pairs who were not currently dieting (Table 3).

In a model that considered both MVPA and energy intake simultaneously, within-pair differences in MVPA remained positively associated with BMI $z$-score differences within overweight-discordant sibling pairs $(\beta=-0.30, \mathrm{p}=0.018$ for 10 min difference in MVPA). Within twin pairs, withinpair differences in energy intake remained associated with differences in BMI z-score ( $\beta=0.11, \mathrm{p}=0.024$ for $100 \mathrm{kcal}$ difference in energy intake).

\section{Discussion}

The present study investigated lifestyle behaviours in relation to BMI differences within same-sex sibling pairs in the I.Family study, as well as overweight-discordant sibling pairs and twin pairs. More time spent in MVPA was consistently associated with lower BMI z-score within all 3 groups of sibling pairs. Higher energy intake was associated with higher BMI z-score within twin pairs, and within sibling pairs who were not currently dieting for weight loss.

Eating and physical activity behaviours can be partly shared by siblings, for example, by parents deciding which foods are available at home or by eating at the same dinner table; however, they may also be to some extent unshared, as 
Table 3 Linear mixed models for the associations of within-pair differences $(\Delta)$ in energy intake (per $100 \mathrm{kcal} / \mathrm{d}$ ) and $\Delta \mathrm{BMI} \mathrm{z}$-score (outcome) within 3 sibling pair groups considering dieting and misreporting.

\begin{tabular}{|c|c|c|c|c|c|}
\hline Independent variables (separate models) & $\mathrm{n}$ & $\beta$ & SE & $95 \% \mathrm{CI}$ & P-value \\
\hline \multicolumn{6}{|c|}{ Model unadjusted for misreporting or dieting status } \\
\hline All sibling pairs & 491 & 0.03 & 0.02 & $0.00,0.06$ & 0.09 \\
\hline Sibling pairs discordant for overweight & 90 & 0.03 & 0.06 & $-0.09,0.15$ & 0.60 \\
\hline Twin pairs $^{\mathrm{a}}$ & 42 & 0.13 & 0.03 & $0.07,0.19$ & $<0.001$ \\
\hline \multicolumn{6}{|l|}{ Model adjusted for dieting status } \\
\hline All sibling pairs & 491 & 0.03 & 0.02 & $0.00,0.06$ & 0.06 \\
\hline Sibling pairs discordant for overweight & 90 & 0.05 & 0.05 & $-0.05,0.15$ & 0.31 \\
\hline Twin pairs $^{\mathrm{a}}$ & 42 & 0.10 & 0.03 & $0.04,0.17$ & 0.002 \\
\hline \multicolumn{6}{|l|}{ Model adjusted for misreporting status } \\
\hline All sibling pairs & 491 & 0.06 & 0.02 & $0.02,0.09$ & 0.001 \\
\hline Sibling pairs discordant for overweight & 90 & 0.14 & 0.05 & $0.04,0.25$ & 0.007 \\
\hline Twin pairs $^{\mathrm{a}}$ & 42 & 0.14 & 0.03 & $0.07,0.20$ & $<0.001$ \\
\hline \multicolumn{6}{|l|}{ Model among sibling pairs who are not dieting } \\
\hline All sibling pairs & 392 & 0.03 & 0.02 & $0.00,0.07$ & 0.046 \\
\hline Sibling pairs discordant for overweight & 50 & 0.06 & 0.06 & $-0.07,0.18$ & 0.37 \\
\hline Twin pairs $^{\mathrm{a}}$ & 35 & 0.10 & 0.02 & $0.06,0.15$ & $<0.001$ \\
\hline \multicolumn{6}{|c|}{ Model among sibling pairs with plausible energy reports } \\
\hline All sibling pairs & 361 & 0.12 & 0.03 & $0.05,0.19$ & 0.001 \\
\hline Sibling pairs discordant for overweight & 63 & 0.20 & 0.11 & $-0.01,0.41$ & 0.062 \\
\hline Twin pairs ${ }^{a}$ & 28 & 0.19 & 0.08 & $0.03,0.35$ & 0.018 \\
\hline
\end{tabular}

All models are adjusting for sex, within-pair differences in age and within-pair differences in height. Country and family are included as random effects, if applicable.

Dieting status of the sibling pairs was coded as " 0 " if both siblings were not dieting, "1" if sibling 1 was dieting, " 2 " if sibling 2 was dieting and " 3 " if both siblings were dieting.

Misredporting of the sibling pairs was coded as " 0 " if energy reports of both siblings were implausible, "1" if sibling 1 was implausible, " 2 " if sibling 2 was implausible and " 3 " if energy reports of both siblings were plausible.

Bold P-values indicate significant at $\mathrm{p}<0.05$.

Abbreviations: BMI, body mass index; $\mathrm{n}$, number of sibling pairs; $\beta$, regression coefficient; $\mathrm{SE}$, standard error; $\mathrm{CI}$, confidence interval.

${ }^{a}$ Including 6 pairs from triplets.

each child has unique experiences that may make children in the same family behave dissimilar to one another. For example, emotional over-eating and under-eating is mainly shaped by the family home environment; however there are also non-shared environmental influence on emotional eating in early childhood [32]. Another example is parenting feeding practices; while parents generally tend to use similar feeding practices with each of their children, they may also have different interactions with each of their children regarding food especially when they are concerned differentially about their children's weight status [33].

In previous observational studies, there is a lack of consistency in research findings that assessed the relationship between energy intake and weight status in children or adolescents [34,35]. More convincing evidence comes from experimental studies of sibling pairs where children with overweight were shown to be prone to overeating as compared to their normal-weight siblings. In a sample of 19 sibling pairs discordant for overweight, Roemmich et al. [36] reported that siblings with overweight consumed greater amounts of energy during a laboratory visit with controlled consumption of a single food. In the same study, sibling differences in accelerometer-derived MVPA predicted differences in BMI $z$-score among children with a mean age of 12 years. In previous experimental studies, it was suggested that siblings with overweight show impaired short-term energy compensation ability and greater susceptibility to eating in the absence of hunger in response to external food cues. In the experiment by Kral et al. [30], siblings with overweight undercompensated and therefore overate after an energydense preload, whereas normal-weight siblings showed more accurate energy compensation. In line with these former experimental studies in siblings, our results show that this does not only apply to short-term energy compensation, but also that sibling pairs that differ in usual energy intake and are not currently on a weight loss diet differ in their BMI $z$-scores. We further show that dieting behaviours are common in children and adolescence, and deliberate underreporting of energy due to dieting needs to be considered in future observational studies of energy intake and BMI.

Of note, energy intake was positively associated with BMI z-score within twin pairs regardless of whether energy misreporting and dieting were considered or not. In contrast to sibling pairs, twin pairs are matched for age and additionally share prenatal factors and special twin environments, and in case of MZ twins they share the same DNA sequence. As twins resemble one another in virtually all human traits and characteristics that can be measured [37], twin pairs are likely more similar to each other than sibling pairs in a whole range of factors that could be confounders in the energy-BMI association (including but not limited to dieting and misreporting behaviour). Ruling out confounding by these additional factors inherently shared by co-twins might explain why the twin comparison design turned out to be a more powerful approach for detecting the energy-BMI 
association than the sibling comparison design. In line with our findings, earlier twin studies among adults have also reported that overweight co-twins eat more [38] and have higher energy intakes (due to higher hunger and disinhibition scores) [39] than normal-weight co-twins.

The selection of appropriate methods to best identify misreporters and to account for them in the analysis is an on-going debate. Some studies have suggested that attempts to lose weight confound the relationship between nutrition behaviours and BMI [40] and controlling for dieting alone reduces the inverse energy intake-BMI association by approximately 20\% [41]. More commonly, studies have suggested to exclude subjects who report implausible energy intakes [42] or statistically adjust for the different reporting groups [43]. In a previous investigation of the IDEFICS sample, Börnhorst et al. [44] compared several statistical approaches to evaluate misreporting of energy and dietary intake in children. While negligence of misreporting masked or even reversed some of the diet-obesity associations, adjustment for the reporting group revealed a positive association between energy intake and overweight/obesity in children [44]. The I.Family study presents the third examination of these IDEFICS children, to which the children's siblings were also invited to participate. This allowed us to examine lifestyle behaviours in relation to BMI within families. The results are consistent with the aforementioned previous IDEFICS investigation and further show that the positive energyBMI association remains even after controlling for familial-level confounding factors, shared pre-natal environmental factors and a larger fraction of genetic factors shared within sibling and twin pairs.

The primary strength of this study is the family recruitment that enabled us to study whether lifestyle behaviours differ among sibling pairs living and growing up in the same household. The novel aspect of the sibling design is that the number of confounding factors is drastically reduced, as this design minimizes family-level confounding factors and therefore improves causal inference compared to cross-sectional study designs of unrelated children. Parental socioeconomic status is an example of an environmental factor that siblings in the same family share. We have chosen sibling pairs close in age to reduce age-related confounding. The siblings with overweight were taller, a finding that is consistent with earlier studies of overweight-discordant sibling [30]. The present study benefits from the availability of a whole range of lifestyle behaviours measured according to a standardized protocol, with partly objective methods, in a considerably larger number of sibling pairs than in previous studies. However, as it was possible to opt out from single examination modules, the actual number of sibling pairs varied for the different lifestyle-related behaviours. This also resulted in a rather small number of siblings that were discordant for overweight. In spite of the lower statistical power in the overweight-discordant pairs, the size of the associations was often larger in discordant siblings than in the analysis including all sibling pairs, probably due to the larger contrast in exposure and outcome. Our data also included a subset of twin pairs of unknown zygosity, allowing us to account for a larger proportion of genetic variation and pregnancy-related factors shared by co-twins. For dietary assessment, we used a computerassisted instrument that included standardized photographs, multiple plausibility checks, and prompting questions that facilitated reporting of accurate portion sizes and complete recalls.

This study is not without limitations. Most importantly, the cross-sectional design does not allow for testing whether the observed lifestyle differences between sibling pairs are causes or consequences of their increased weight. We acknowledge the absence of genotype data. Differences within sibling pairs for quite heritable traits such as BMI [7] may be explained by unique environmental experiences or genetic variation - in our study it is impossible to distinguish between the two owing the absence of genetic data. While misreporting is present in all self-report dietary assessment tools, multiple 24 -h recalls provide better estimates of absolute dietary intakes than FFQs [45]. In general, our results support the use of measurement error corrections for recall-based methods to ascertain usual energy intake, but it must be acknowledged that children and adolescents present a special challenge. The use of body weight-dependent equations to estimate energy requirements (such as the Goldberg method) could have overestimated the effect size for the association of energy intake and BMI as a result of selection bias [46]. Thus, correcting for external predictors of misreporting such as dietary restrained has been recommended as an alternative approach to account for misreporting when BMI is the outcome variable [47]. Thus, we also analysed the energyBMI association stratified by dieting status of the sibling pairs and found that energy intake and BMI z-score were positively associated within sibling pairs who were not currently dieting for weight loss.

In conclusion, MVPA was the behaviour most consistently associated with BMI differences within sibling pairs growing up in the same home. Energy intake was positively associated with BMI z-score differences within twin pairs and within all sibling pairs who were not dieting for weight loss. This finding is consistent with previous sibling studies on short-term energy regulation, but has so far proven difficult to show in observational studies of children. Thus, dieting behaviours need to be considered when examining the relationship between energy intake and BMI in future observational studies of children and adolescence. Future studies should further explore whether genetic variants regulating appetite or energy expenditure behaviours distinguish sibling pairs that are discordant for overweight or obesity.

\section{Acknowledgements}

We thank the I.Family children and their parents for participating in this extensive examination. We are grateful for the support from school boards, headmasters and communities. We also express our gratitude to the entire I.Family study teams, i.e. our study nurses and 
interviewers, intervention managers, student assistants, IT personnel, data managers, laboratory technicians, administrative staff, paediatricians and researchers.

\section{Ethics approval and consent to participate}

We certify that all applicable institutional and governmental regulations concerning the ethical use of human volunteers were followed during this research. Ethics approval was obtained from responsible committees in each country in accordance with the ethical standards of the 1964 Declaration of Helsinki and its later amendments. All parents or legal guardians of the participating minor children gave written informed consent. Children older than 16 years provided written informed consent, while children aged 12 and over gave a simplified written consent. Younger children gave oral consent for examinations and sample collection.

\section{Funding}

This work was done as part of the I.Family Study (http:// www.ifamilystudy.eu/). We gratefully acknowledge the financial support of the European Community within the Seventh RTD Framework Programme Contract No. 266044. The lead author also wishes to thank the Juho Vainio Foundation, the Yrjö Jahnsson Foundation and the German Academic Exchange Service (DAAD) for their financial support.

\section{Conflict of interest}

The authors declare that they have no conflict of interest to report.

\section{Authors' contributions}

LHB, JK, KM, LL and AH conceived the study and/or contributed to the planning and design of the study. LHB, GM, TI and KM contributed to processing of the data and/ or statistical analyses. LHB wrote the manuscript. PR, NM, VP, DM, MT, TV, LM, WA, LL and AH made substantial contributions to the acquisition of data and/or were involved in revising the manuscript for important intellectual content. JK, KM, LJ, AK-R and LR made contributions to drafting the manuscript. All authors critically reviewed the manuscript and had final approval of the submitted version.

\section{Availability of data and materials}

Due to the high sensitive data collected in children, ethical restrictions prohibit the authors from making the minimal data set publicly available. Data are however available from the authors upon reasonable request and with permission of the Steering Committee on a case-by-case basis. Interested researchers can contact the I.Family consortium (http://www.ifamilystudy.eu/) or the study coordinator (Ahrens@leibniz-bips.de) to discuss possibilities for data access.

\section{Consent for publication}

Not applicable.

\section{References}

[1] Weiss R, Dziura J, Burgert TS, Tamborlane WV, Taksali SE, Yeckel CW, et al. Obesity and the metabolic syndrome in children and adolescents. N Engl J Med 2004;350(23):2362-74.

[2] Puder JJ, Munsch S. Psychological correlates of childhood obesity. Int J Obes 2010;34(Suppl. 2):S37-43.

[3] Lloyd LJ, Langley-Evans SC, McMullen S. Childhood obesity and adult cardiovascular disease risk: a systematic review. Int J Obes 2010;34(1):18-28.

[4] Magnusson PK, Rasmussen F. Familial resemblance of body mass index and familial risk of high and low body mass index. A study of young men in Sweden. Int J Obes Relat Metab Disord 2002;26(9): 1225-31.

[5] Nakamura S, Narimatsu H, Sato H, Sho R, Otani K, Kawasaki R, et al. Gene-environment interactions in obesity: implication for future applications in preventive medicine. J Hum Genet 2016;61(4): $317-22$.

[6] Silventoinen K, Jelenkovic A, Sund R, Hur YM, Yokoyama Y, Honda C, et al. Genetic and environmental effects on body mass index from infancy to the onset of adulthood: an individual-based pooled analysis of 45 twin cohorts participating in the COllaborative project of Development of Anthropometrical measures in Twins (CODATwins) study. Am J Clin Nutr 2016;104(2):371-9.

[7] Elks CE, den Hoed M, Zhao JH, Sharp SJ, Wareham NJ, Loos RJ, et al. Variability in the heritability of body mass index: a systematic review and meta-regression. Front Endocrinol 2012;3:29.

[8] Katzmarzyk PT, Barreira TV, Broyles ST, Champagne CM, Chaput JP, Fogelholm M, et al. Relationship between lifestyle behaviors and obesity in children ages 9-11: results from a 12-country study. Obesity 2015;23(8):1696-702.

[9] Hebestreit A, Barba G, De Henauw S, Eiben G, Hadjigeorgiou C, Kovács É, et al. Cross-sectional and longitudinal associations between energy intake and BMI z-score in European children. Int J Behav Nutr Phys Act 2016;13:23.

[10] Hense S, Pohlabeln H, De Henauw S, Eiben G, Molnar D, Moreno LA, et al. Sleep duration and overweight in European children: is the association modified by geographic region? Sleep 2011;34(7):885-90.

[11] Lissner L, Lanfer A, Gwozdz W, Olafsdottir S, Eiben G, Moreno LA, et al. Television habits in relation to overweight, diet and taste preferences in European children: the IDEFICS study. Eur J Epidemiol 2012;27(9):705-15.

[12] Donatiello E, Dello Russo M, Formisano A, Lauria F, Nappo A, Reineke A, et al. Physical activity, adiposity and urbanization level in children: results for the Italian cohort of the IDEFICS study. Publ Health 2013;127(8):761-5.

[13] Susser E, Eide MG, Begg M. Invited commentary: the use of sibship studies to detect familial confounding. Am J Epidemiol 2010; 172(5):537-9.

[14] Ahrens W, Siani A, Adan R, De Henauw S, Eiben G, Gwozdz W, et al. Cohort Profile: the transition from childhood to adolescence in European children-how I.Family extends the IDEFICS cohort. Int J Epidemiol 2017;46(5):1394-1395j.

[15] Stomfai S, Ahrens W, Bammann K, Kovács E, Mårild S, Michels N, et al. Intra- and inter-observer reliability in anthropometric measurements in children. Int J Obes 2011;35(Suppl. 1):S45-51.

[16] Cole TJ, Freeman JV, Preece MA. British 1990 growth reference centiles for weight, height, body mass index and head circumference fitted by maximum penalized likelihood. Stat Med 1998; 17(4):407-29. 
[17] Cole T], Lobstein T. Extended international (IOTF) body mass index cut-offs for thinness, overweight and obesity. Pediatr Obes 2012; 7(4):284-94.

[18] Konstabel K, Veidebaum T, Verbestel V, Moreno LA, Bammann K, Tornaritis M, et al. Objectively measured physical activity in European children: the IDEFICS study. Int J Obes 2014;38(Suppl. 2): S135-43.

[19] Evenson KR, Catellier DJ, Gill K, Ondrak KS, McMurray RG. Calibration of two objective measures of physical activity for children. J Sports Sci 2008;26(14):1557-65.

[20] Intemann T, Pigeot I, De Henauw S, Eiben G, Lissner L, Krogh V, et al. Urinary sucrose and fructose to validate self-reported sugar intake in children and adolescents: results from the I.Family study. Eur J Nutr 2018 Mar 6. https://doi.org/10.1007/s00394-018-1649-6 [Epub ahead of print].

[21] Hebestreit A, Wolters M, Jilani H, Eiben G, Pala V. Web-based 24hour dietary recall: the SACANA program. Instruments for health surveys in children and adolescents, 1 st. New York, Dordrecht, Heidelberg, London: Springer; 2018.

[22] Börnhorst C, Huybrechts I, Hebestreit A, Krogh V, De Decker A, Barba $G$, et al. Usual energy and macronutrient intakes in 2-9year-old European children. Int J Obes 2014;38(Suppl. 2): S115-23.

[23] Hebestreit A, Börnhorst C, Pala V, Barba G, Eiben G, Veidebaum T, et al. Dietary energy density in young children across Europe. Int J Obes 2014;38(Suppl. 2):S124-34.

[24] Livingstone MB, Robson PJ. Measurement of dietary intake in children. Proc Nutr Soc 2000;59(2):279-93.

[25] Goldberg GR, Black AE, Jebb SA, Cole TJ, Murgatroyd PR, Coward WA, et al. Critical evaluation of energy intake data using fundamental principles of energy physiology: 1. Derivation of cutoff limits to identify under-recording. Eur J Clin Nutr 1991;45(12): 569-81.

[26] Börnhorst C, Huybrechts I, Ahrens W, Eiben G, Michels N, Pala V, et al. Prevalence and determinants of misreporting among European children in proxy-reported 24 h dietary recalls. Br J Nutr 2013;109(7):1257-65.

[27] Tooze JA, Kipnis V, Buckman DW, Carroll RJ, Freedman LS, Guenther PM, et al. A mixed-effects model approach for estimating the distribution of usual intake of nutrients: the $\mathrm{NCI}$ method. Stat Med 2010;29(27):2857-68.

[28] Kipnis V, Midthune D, Buckman DW, Dodd KW, Guenther PM, Krebs-Smith SM, et al. Modeling data with excess zeros and measurement error: application to evaluating relationships between episodically consumed foods and health outcomes. Biometrics 2009;65(4):1003-10.

[29] Hebestreit A, Intemann T, Siani A, De Henauw S, Eiben G, Kourides YA, et al. Dietary patterns of European children and their parents in association with family food environment: results from the I.Family Study. Nutrients 2017;9:17.

[30] Kral TV, Allison DB, Birch LL, Stallings VA, Moore RH, Faith MS. Caloric compensation and eating in the absence of hunger in 5- to 12-y-old weight-discordant siblings. Am J Clin Nutr 2012;96(3): 574-83.

[31] Salvy SJ, Feda DM, Epstein LH, Roemmich JN. Friends and social contexts as unshared environments: a discordant sibling analysis of obesity- and health-related behaviors in young adolescents. Int J Obes 2017;41(4):569-75.

[32] Herle M, Fildes A, Llewellyn $\mathrm{CH}$. Emotional eating is learned not inherited in children, regardless of obesity risk. Pediatr Obes. 2018
Oct;13(10):628-31. https://doi.org/10.1111/ijpo.12428 [Epub 2018 Jun 21].

[33] Payne LO, Galloway AT, Webb RM. Parental use of differential restrictive feeding practices with siblings. Int J Pediatr Obes 2011; 6(2-2):e540-6.

[34] Anderson YC, Wynter LE, Butler MS, Grant CC, Stewart JM, Cave TL, et al. Dietary intake and eating behaviours of obese New Zealand children and adolescents enrolled in a community-based intervention Programme. PLoS One 2016;11(11):e0166996.

[35] Rodriguez G, Moreno LA. Is dietary intake able to explain differences in body fatness in children and adolescents? Nutr Metabol Cardiovasc Dis 2006;16(4):294-301.

[36] Roemmich JN, White TM, Paluch R, Epstein LH. Energy intake, parental control of children's eating, and physical activity in siblings discordant for adiposity. Appetite 2010;55(2):325-31.

[37] Polderman T], Benyamin B, de Leeuw CA, Sullivan PF, van Bochoven A, Visscher PM, et al. Meta-analysis of the heritability of human traits based on fifty years of twin studies. Nat Genet 2015; 47(7):702-9.

[38] Pietiläinen KH, Korkeila M, Bogl LH, Westerterp KR, YkiJärvinen $\mathrm{H}$, Kaprio J, et al. Inaccuracies in food and physical activity diaries of obese subjects: complementary evidence from doubly labeled water and co-twin assessments. Int J Obes 2010; 34(3):437-45.

[39] Hakala P, Rissanen A, Koskenvuo M, Kaprio J, Ronnemaa T. Environmental factors in the development of obesity in identical twins. Int J Obes Relat Metab Disord 1999;23(7):746-53.

[40] Utter J, Scragg R, Ni Mhurchu C, Schaaf D. What effect do attempts to lose weight have on the observed relationship between nutrition behaviors and body mass index among adolescents? Int J Behav Nutr Phys Act 2007;4. 40-40.

[41] Ballard-Barbash R, Graubard I, Krebs-Smith SM, Schatzkin A, Thompson FE. Contribution of dieting to the inverse association between energy intake and body mass index. Eur J Clin Nutr 1996; 50(2):98-106.

[42] Huang TT, Roberts SB, Howarth NC, McCrory MA. Effect of screening out implausible energy intake reports on relationships between diet and BMI. Obes Res 2005;13(7):1205-17.

[43] Tooze JA, Freedman LS, Carroll RJ, Midthune D, Kipnis V. The impact of stratification by implausible energy reporting status on estimates of diet-health relationships. Biom J Biom Z 2016;58(6): $1538-51$.

[44] Börnhorst C, Huybrechts I, Hebestreit A, Vanaelst B, Molnár D, BelSerrat S, et al. Diet-obesity associations in children: approaches to counteract attenuation caused by misreporting. Publ Health Nutr 2013;16(2):256-66.

[45] Park Y, Dodd KW, Kipnis V, Thompson FE, Potischman N, Schoeller DA, et al. Comparison of self-reported dietary intakes from the Automated Self-Administered 24-h recall, 4-d food records, and food-frequency questionnaires against recovery biomarkers. Am J Clin Nutr 2018;107(1):80-93.

[46] Rhee JJ, Sampson L, Cho E, Hughes MD, Hu FB, Willett WC. Comparison of methods to account for implausible reporting of energy intake in epidemiologic studies. Am J Epidemiol 2015;181(4): 225-33.

[47] Vainik U, Konstabel K, Latt E, Maestu J, Purge P, Jurimae J. Diet misreporting can be corrected: confirmation of the association between energy intake and fat-free mass in adolescents. Br J Nutr 2016;116(8):1425-36. 